\title{
Carrier relaxation in quantum wires: consequences for quantum wire laser performance
}

\author{
Igor Vurgaftman and Jasprit Singh \\ Solid State Electronics Laboratory, Department of Electrical Engineering and \\ Computer Science, The University of Michigan, Ann Arbor, Ml 48109-2122, USA
}

\begin{abstract}
Quantum wire lasers are expected to require very low threshold currents owing to the nature of the TD density of states which develops a sharp peak at the band edge and ensures superior laser characteristics. However, carrier relaxation processes in quasi-1D structures may be much slower than in bulk material owing to reduction in the momentum space. For very long relaxation times, these equilibrium processes are expected to limit the maximum modulation frequency of the quantum wire lasers. We perform a Monte Carlo simulation of electron relaxation in quantum wires with the inclusion of the electron-bulklike polar optical and acoustic phonon, electron-electron and electron-hole interactions as well as Thomas-Fermi screening. We find that for a carrier density of $10^{18} \mathrm{~cm}^{-3}$, the electron relaxation time ranges from $120 \mathrm{ps}$ for the

$100 \AA \times 100 \AA$ wire to 30 ps for the $200 \AA \times 200 \AA$ wire. Since the threshold current in a quantum wire laser increases with the wire cross section, within the limits of our relaxation model, this indicates possible existence of a trade-off between speed and efficiency in a quantum wire laser. We also analyse the effects of carrier relaxation on gain compression in quantum wire lasers by solving the Boltzmann equation using a novel Monte Carlo technique. A spectral hole forms in the carrier distribution at high injected currents with the resulting decrease in the slope of the light-current characteristic. The effect of a non-Fermi-Dirac distribution of electrons is found to result in a suppression of the peak gain as compared with the peak gain calculated using the equilibrium distribution.
\end{abstract}

In recent years, considerable attention has been devoted to the applications of quasi-1D electronic structures in constructing very low-threshold and very high-speed semiconductor lasers. The improvement in threshold current and speed is expected to stem from the sharp peak in the density of states that occurs near the band edge in quantum wire structures $[1,2]$. However, it should be kept in mind that the reduction in the momentum space to one dimension leads to qualitatively new phenomena in the carrier relaxation process which may increase the equilibration time to such an extent that high-speed operation of quantum wire lasers can be made problematic $[3,4]$. Intersubband electron-electron scattering is prohibited, and thermalization of the electron gas, which loses its energy primarily by emitting monoenergetic polar optical phonons (POP), is exceedingly slow, leading to bottlenecks in the relaxation process [4]. Thermalization in the photoexcited electron gas with the inclusion of electron-electron and polar optical phonon scattering has been studied [5], but relaxation of carriers injected into the active region and also undergoing acoustic phonon and electron-hole scattering events has not been investigated to the best of our knowledge. Here we study quantitatively the physics of the equilibration process using the Monte Carlo simulation technique and then proceed to examine the consequences of the slow carrier relaxation processes for the performance of quantum wire lasers. We consider in particular the effect of electron relaxation on the nonlinear gain characteristics and present qualitative results for gain compression due to spectral hole burning.

The relaxation processes in the valence band are expected to be much faster than those in the conduction band owing to the higher valence band density of states; therefore, in this paper we are concerned solely with electron relaxation. We simulate the relaxation process by injecting electrons in a thermal distribution at the potential well edge and following their progress as they come to thermal equilibrium with the lattice maintained at a constant temperature of $300 \mathrm{~K}$. The conduction band 1D states are calculated in the effective mass approximation by numerically discretizing Schrödinger's equation on a uniform mesh in the wire region and converting derivatives into finite differences, and solving the resulting matrix equation iteratively. For the $100 \AA \times 100 \AA$ wire, two subbands lie below the potential well, the first one 
at $0.05 \mathrm{eV}$ and the second one at $0.11 \mathrm{eV}$, while there are six subbands below the conduction band discontinuity in the $200 \AA \times 200 \AA$ wire. The valence band states are found by diagonalizing the four-band Kohn-Luttinger Hamiltonian by the same finite difference approach.

The major energy loss process is found to be emission of polar optical phonons. The only intersubband electronelectron scattering process allowed by the requirement of simultaneous conservation of energy and momentum in a quasi-1D system is an exchange of states between the interacting electrons. This scattering process cannot alter the shape of the distribution function. In the virtual absence of electron-electron scattering, it becomes necessary to include inelastic acoustic phonon scattering as well as electron-hole scattering into the Monte Carlo simulation in order to describe the thermalization processes in a quantum wire. Since we consider quantum wires with cross sections from $100 \AA \times 100 \AA$ up, we assume that the coupling between the confined electronic states and localized interface phonon modes is much weaker than that between electrons and bulk-like confined phonon modes and include only the latter interaction in our calculations. The coupling coefficient for the POP scattering is obtained from the Fröhlich Hamiltonian [6], and that for AP (acoustic phonon) scattering from the deformation potential theory. The details of the simulation have been presented elsewhere [4], and the full formalism is forthcoming [7].

In order to estimate the effect of electron-hole scattering on the evolution of the electron distribution, we adopt the assumption of the equilibrium hole distribution because the full relaxation problem involving two types of carriers is too complex to be solved in practical simulation times. Moreover, the hole-phonon coupling is expected to be significantly stronger than the eiectron-phonon coupling with the rate of energy loss for holes far greater than that for electrons. We also approximate the valence band structure as parabolic, which is equivalent to setting the off-diagonal terms of the Kohn-Luttinger Hamiltonian to zero. This assumption results in a major simplification of the calculational technique while the consequences for the electron-hole scattering processes are believed to be secondary. Electrons and holes interact through the Coulombic attraction, and the scattering rate can be computed most easily in the Born approximation [8]. Screening effects are incorporated in the static longwave limit.

The average energy of an ensemble of 5000 electrons as a function of time after injection is shown in figure 1 for the $100 \AA \times 100 \AA \mathrm{GaAs} / \mathrm{Al}_{0.3} \mathrm{Ga}_{0.7}$ wire for two carrier concentrations. While the initial stage in the electron relaxation process can be modelled as exponential decay with a characteristic time of several picoseconds, the rate of energy loss is reduced drastically after $\approx 10 \mathrm{ps}$. This phenomenon can be accounted for by the fact that the carriers within the pop energy ( $36 \mathrm{meV}$ ) of the effective band edge can give up energy only by emitting acoustic phonons, which is a statistically very slow process. Note that the multiple subband occupation increases the mean energy for the equilibrium distribution

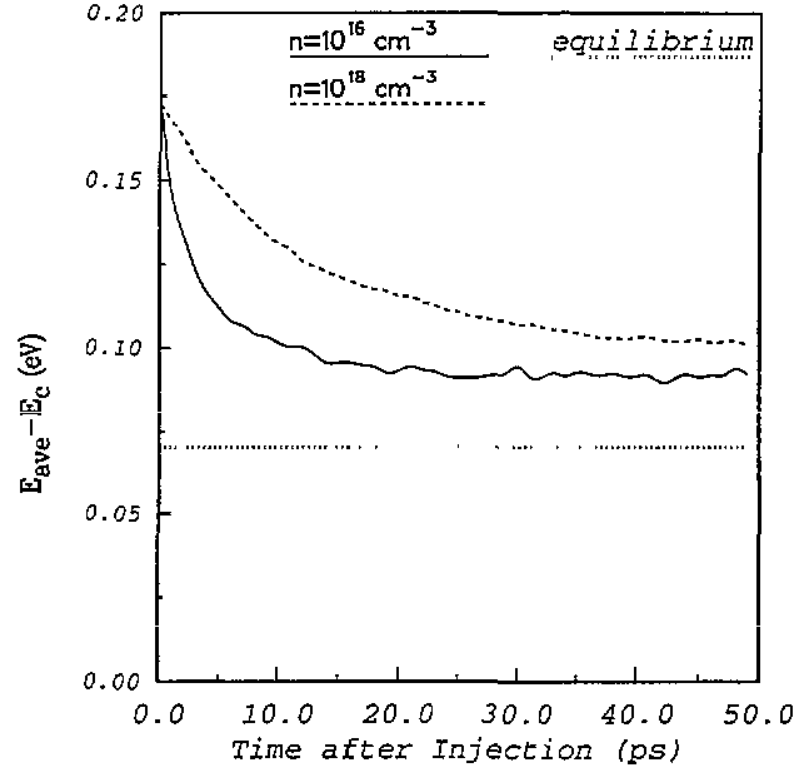

Figure 1. The average energy for an ensemble of 5000 electrons as a function of time afiter injection for a $100 \AA \times 100 \AA$ GaAs quantum wire for two carrier concentrations with and without electron-hole scattering. The horizontal line corresponds to the mean energy for the equilibrium Fermi-Dirac distribution.

above the strictly $1 \mathrm{D} k_{\mathrm{B}} T / 2$ value. The results of the Monte Carlo simulation indicate that after $10 \mathrm{ps}$ the majority of electrons are involved in transitions between the first two subbands in the wire region.

Also shown in figure 1 are the results of the Monte Carlo simulation with the inclusion of electron-hole scattering, which allows partial thermalization of the electron distribution as well as a certain rate of energy loss because of the assumption of an equilibrium hole distribution. The energy relaxation of the electron distribution proceeds at a faster rate than in the absence of the electron-hole interaction. The bottleneck is still present, but it can be overcome sooner owing to the loss of energy by the electrons to the 'cool' equilibriunin hole distribution. This indicates the importance of including electron-hole scattering in relaxation time calculations for low-dimensional systems. More comprehensive models may include hot-hole effects and non-parabolicity in the valence band provided an efficient computational procedure can be found.

Nonlinear gain effects in semiconductor lasers have been previously treated using the density matrix formalism and third-order perturbation theory $[9,10]$. The drawback of this approach is that the carrier relaxation effects are introduced in a phenomenological manner through the intraband relaxation time. We have been able to formulate a technique that allows one to calculate the exact form of the distribution function and the gain spectrum with the inclusion of recombination and injection effects from a knowledge of the band structure and scattering rates in a semiconductor laser. The method is applied to examine the phneomenon of spectral hole burning in quantum wire lasers. We start from the 
Boltzmann equation in a homogeneous medium with no applied fields

$$
\begin{aligned}
\frac{\partial f(E)}{\partial t} & =f(E+\hbar \omega)(1-f(E)) W_{\mathrm{cms}}(E+\hbar \omega, E) \\
& +f(E-\hbar \omega)(1-f(E)) W_{\mathrm{abs}}(E-\hbar \omega, E) \\
& -f(E)[1-f(E+\hbar \omega)] W_{\mathrm{abs}}(E, E+\hbar \omega) \\
& -f(E)[1-f(E-\hbar \omega)] W_{\mathrm{cms}}(E, E-\hbar \omega)
\end{aligned}
$$

where $W_{\text {abs }}$ and $W_{\text {emis }}$ represent the scattering rates for emission and absorption of phonons for all $k$-states in the corresponding energy intervals. The time derivative is set to zero in order to solve for the steady-state distribution function. The equation has an infinite number of solutions, of which the Fermi-Dirac distribution is the one to which the electron distribution is driven in the long run under the influence of thermalization processes, such as electron-hole scattering in quantum wires. The effect of stimulated emission at the lasing frequency and carrier injection at the edge of the potential well formed by the barrier region is now taken into account by introducing the source $(1-f(E)) / \tau$ and sink $-f(E) / \tau$ terms. The energy spectrum of interest is then subdivided into small intervals, and the Boltzmann equation is converted into a set of coupled nonlinear equations. The equations are greatly simplified if only POP scattering is included, because only points separated by multiples of the POP energy are coupled. The AP scattering is then treated as contributing a broadening of the solution of the simplified equation with the linewidth proportional to the AP scattering rate. This is an adequate approximation since Pop scattering rates are greater than AP scattering rates in quantum wires by at least an order of magnitude.

The direct numerical solution of the resulting system of nonlinear coupled equations is cumbersome if a fne mesh on the energy spectrum is set up and excellent convergence is desired. Instead we solve the problem by a Monte Carlo technique. We start with the Fermi-Dirac distribution function and generate random perturbations accepting only those changes which decrease the time derivative of the distribution function in each energy interval. For characteristic injection times between $10 \mathrm{ps}$ and $1 \mathrm{~ns}$ we find that the solution rapidly converges to the steady-state distribution.

The steady-state distribution function for a characteristic time of $10 \mathrm{ps}$ is shown in figure 2 . This recombination time roughly corresponds to a current density 100 times the threshold current density calculated for the $100 \AA \times 100 \AA$ wire. The effect of injection and extraction terms on the distribution function is seen to be twofold: a spectral hole is burned around the lasing wavelength and the electron gas effective temperature is raised. The magnitude of gain compression can be estimated by calculating the material gain from the Fermi golden rule. We substitute the distribution function obtained with the inclusion of spectral hole burning effects. The result is shown in figure 3. Mimicking the singularity in the joint density of states, the gain spectrum is sharply peaked

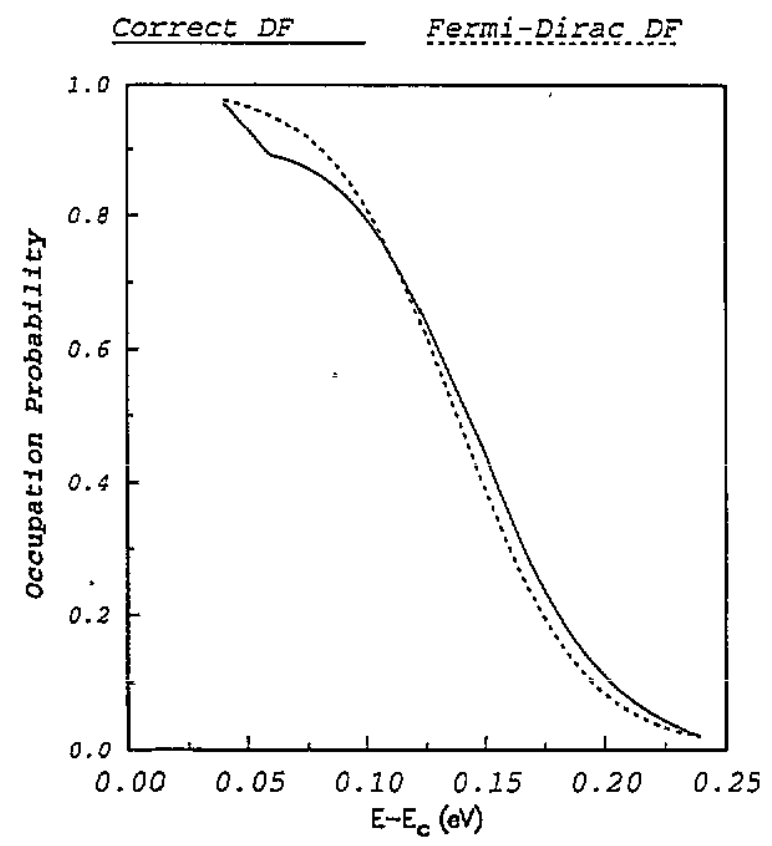

Figure 2. The distribution function in the presence of spectral hoie burning is shown by the fuli curve. For comparison, the equilibrium Fermi-Dirac distribution function is given by the broken curve. The characteristic injection and extraction time is $10 \mathrm{ps,}$, approximately corresponding to a current density 100 times that at threshold.

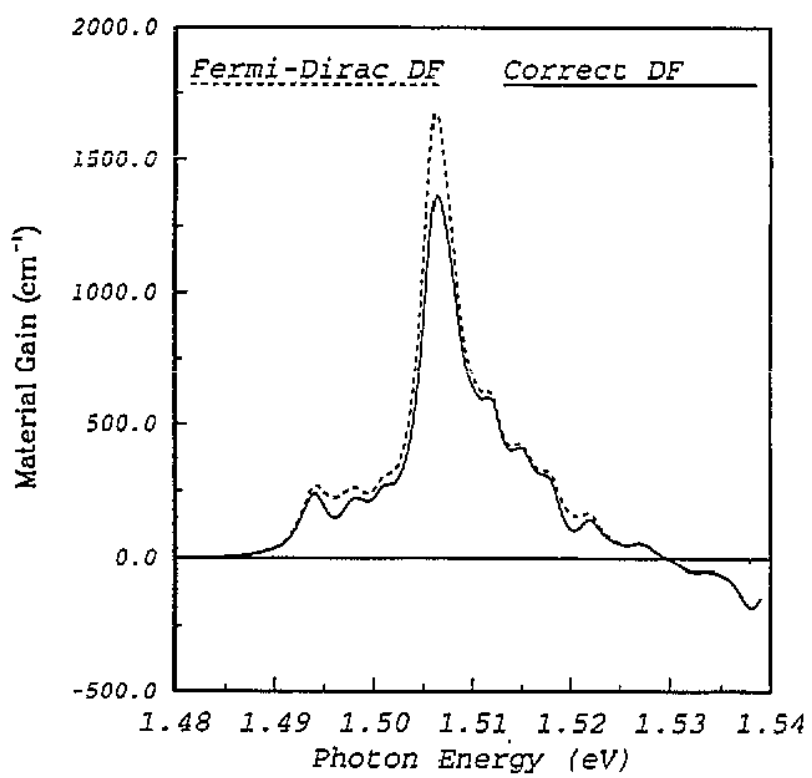

Figure 3. Gain compression resilting from the inclusion of spectral hole burning. The full curve represents the material gain spectrum obtained by using the correct distribution, while the broken curve represents that obtained using the Fermi-Dirac distribution. The characteristic injection and extraction time is $10 \mathrm{ps,}$ approximately corresponding to a current density 100 times that at threshold.

around the lasing frequency, and the nonlinear gain effects are clearly seen to amount to the suppression of the peak gain. Note that, in the context of this paper, we refer to the spectral hole formed in the distribution function rather than in the gain spectrum as the "hole 
burning' effect. No hole in the gain spectrum is observed because of the assumption of single-mode lasing and the sharpness of the gain curve.

To summarize, we have considered the effects of a finite relaxation time in quantum wires on the performance characteristics of quantum wire lasers. The importance of the electron-hole scattering processes has been brought out by our studies. Although electron-hole scattering does speed up carrier thermalization, the relaxation rates are still quite low. Besides limiting the intrinsic modulation bandwidth of the quantum wire laser, the increased relaxation time is found to aggravate the nonlinear gain effects in a quantum wire laser. We have calculated electron relaxation times in quantum wire structures by a Monte Carlo simulation as well as gain compression resulting from the spectral hole burned in the electron distribution function using a novel Monte Carlo technique of solving the Boltzmann equation. Our method of accounting for the nonlinear gain effects in quantum wires is particulariy useful because it provides a straightforward yet fuliy consistent computational technique and can be generalized easily to multimode operation and various scattering processes.

\section{Acknowledgment}

This work was supported by the US Army's URI program (grant no DAAL 03-92-G-0109).

\section{References}

[1] Arakawa Y and Sakaki H 1982 Appl. Phys. Lett. 40 939

[2] Arakawa Y, Vahaia K and Yariv À 1984 Appi. Phys. Lett. 45950

[3] Leburton J P 1992 Phys. Rev. B 4511022

[4] Vurgaftman I and Singh J 1993 Appl. Phys. Lett. 62 2251

[5] Rota L, Rossi F, Goodnick S M, Lugli P, Molinari E and Porod W 1993 Phys. Rev. B 471632

[6] Leburton J P 1984 J. Appl. Phys. 562850

[7] Vurgaftman I and Singh J 1994 IEEE J. Quantum Electron. to be published

[8] Goodnick S M and Lugli P 1988 Phys. Rev. B 37 2578

[9] Asada $M$ and Suematsu Y 1985 IEEE J. Quantum Electron. 21434

[10] Agrawal G P 1987 IEEE J. Quantum Electron. 23 860 